\title{
A Novel Algorithm for Exemplar-based Image Inpainting
}

\author{
Yaru Cheng, Weibin Liu * \\ Institute of Information Science \\ Beijing Jiaotong University \\ Beijing 100044, China
}

Corresponding author: Weibin Liu,wbliu@bjtu.edu.cn

\author{
Weiwei Xing \\ School of Software Engineering \\ Beijing Jiaotong University \\ Beijing 100044, China
}

\begin{abstract}
In traditional exemplar-based image inpainting algorithm, the confidence value will rapidly decrease to zero as the inpainting process progresses. As a consequence, it will lead to unreliable result of the priority calculation and wrong direction of the process. In addition, traditional methods usually use the sum of squared differences (SSD) criterion to search the optimal matching block. Since the matching criterion is single and the precision is limited, the process is easy to produce mismatch. In order to solve the above problems, an improved algorithm has been proposed in this paper. First, we proposed a new confidence update algorithm through replacing the previous linear function form by using a logarithmic function form, which can suppress the phenomenon that the confidence attenuation is too fast and improve the accuracy of guiding and inpainting direction. Then, we combine the physical distance between blocks and traditional SSD matching criterion to improve matching accuracy. The experimental results show that the algorithm overcomes the shortcomings of the traditional algorithm and provides higher quality image restoration effects and better visual effects.
\end{abstract}

Keywords-Exemplar; image inpainting; matching accuracy; Confidence term update

\section{INTRODUCTION}

Digital image inpainting technology has broad application prospects and is a prerequisite for image compression, image enhancement, image recognition and other technologies [1]. First, it need to research and solve how to better detect the damaged part of the image. Then, image inpainting algorithm can automatically inpaint the image according to the known information around the damaged area. Therefore, the research and exploration of image inpainting technology is a subject including both theoretical and practical value [2]. In the time, the requirements for images and video quality around us become higher and higher [3]. Therefore, it is particularly important to research on digital image inpainting.

Recently, many researchers have proposed effective image inpainting models. According to the inpainting theories, they can be devided into two categories: geometry based approaches and exemplar-based approaches [5]. Image inpainting techniques based on non-texture structures are often used to inpaint images with small damaged areas, such as scratch repair and text contamination repair [6]. In 2000, Bertalmio et.al proposed BSCB model based on partial differential equation image inpainting [7]. The main idea of this model is to extending the isophote line of the damaged region's boundary and propagating

\{Correspending author: Weibin Liu, wbliu@bjtu.edu.cn\}

DOI reference number: 10.18293/SEKE2019-152 it into the damaged area. Although the BSCB model has a good inpainting result, it is based on the diffusion and transmission of the inpainting process. Objectively, it is very difficult to carry out mathematical analysis. Chan and Shen proposed the total variational model (TV model) [8] and the curvature-driven diffusion model (CDD model) [9] based on the BSCS model. Then, Telea proposed a fast matching algorithm (FMM) [10].

In 2004, Criminisi proposed the exemplar-based inpainting algorithm, which is on patch level [11]-[12]. Then, many exemplar-based image inpainting methods have emerged [13] and they have been widely used. In [14], Barnes proposed a new image editing algorithm PatchMatch to find approximate nearest-neighbor patches from image patches. This interactive technique has been used by Adobe photoshop because of the flexibility, convenience and simplicity. In [15], Liu used multiscale graph cuts in the process. In [16], Komodakis used MRF-based global optimization method to solve the inpainting problem, which is known as priority belief propagation (p-BP). There are some other researchers do some work based on priority belief propagation [17]-[18]. Then, Ruzic introduced contextaware patch-based image inpainting, where candidate patches are searched over the entire source region based on contextual similarity [19]. The author found a new priority calculation in [20] and make up for the shortcomings in Criminisi method. In [21], they used Pixel Inhomogeneity Factor (PIF) to replace the data term. In [22], Huang use the planar structure guidance to solve the inpainting problems. In [23], Deng separated the confidence term and data term, then he proposed an automatic algorithm to estimate steps of the new priority definition.

This paper proposed a novel algorithm for exemplar-based inpainting method. The main improvement is to change the matching criteria and confidence update function. First, we propose an improved confidence update function to maximize the accuracy of the guided inpainting direction. Then, we combine the physical distance between blocks and traditional SSD matching criterion to search the best matching patch. Novel method overcomes the drawback of Criminisi algorithm in visual inconsistency. We compare the method with two methods in some structure and texture pictures.

\section{RELATED WORK}

In this section, we mainly introduce the exemplar based inpainting method [12]. First, the image is divided into two parts: the target region is defined as the area which is to be inpainted, 
using $\Omega$ to represent. The left area is called source region $\Phi$. Additionally, $\delta \Omega$ represents the boundary of the target region. The inpainting task is to fill in the target region $\Omega$ using the image information from the source region $\Phi$. The inpainting process is shown in Fig.1.

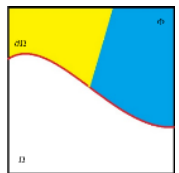

(a)

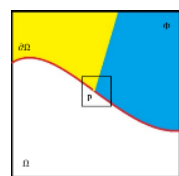

(b)

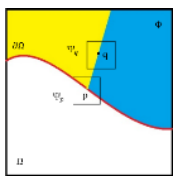

(c)

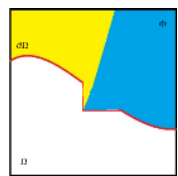

(d)
Fig.1 Inpainting process by Criminisi Method [12]

\section{A. Deciding the filling order}

This step is mainly aimed to determine the filling order of the patch in the target region. For each pixel $p$ along the target region's boundary, we set a square patch $\varphi_{p}$ which is centered on the pixel. In our paper, we set the patch size with $9 \times 9$ pixels throughout the process. After computing the priorities of all pixels along the boundary, we choose the pixel $p$ with the highest priority as the center pixel, then the patch $\varphi_{p}$ will be the target patch to be inpainted.

In Criminisi method [12], the filling priority is defined as follows:

$$
P_{(p)}=C_{(p)} D_{(p)}
$$

Where $C_{(p)}$ represents the confidence term and $D_{(p)}$ represents the data term and theyare calculated by the following equations:

$$
\begin{gathered}
C_{(p)}=\frac{\sum_{q \epsilon \varphi_{p} \cap \bar{\Omega}} C_{(q)}}{\left|\varphi_{p}\right|}, 0 \leq C_{(p)} \leq 1 \\
D_{(p)}=\frac{\left|\nabla I_{p}^{\perp} \cdot n_{p}\right|}{\alpha}, \quad 0 \leq D_{(p)} \leq 1
\end{gathered}
$$

In equation (2), $\bar{\Omega}$ denotes the complementary set of target region $\Omega,\left|\varphi_{p}\right|$ represents the aere of the patch $\varphi_{p}$. During initialization, the function $C_{(p)}$ is set to

$$
\begin{array}{ll}
C_{(p)}=0, & \forall p \in \Omega \\
C_{(p)}=1, & \forall p \in \bar{\Omega}
\end{array}
$$

For each pixel $p$ on the boundary of target region, the confidence term $C_{(p)}$ equals to the ratio between the sum of pixels confidence in $\varphi_{p} \cap \bar{\Omega}$ and the total number of nonzero elements in $\varphi_{p}$. The higher of $C_{(p)}$, the more reliable information surrounding $p$. The intention is that we can inpaint the patch with more reliable information firstly.

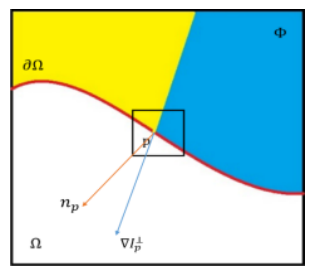

Fig. 2 The iron of $n_{p}$ and $\nabla I_{p}^{\perp}$

Similarly, $n_{p}$ is the unit vector orthogonal to the front $\delta \Omega$ in the pixel $p$ and $\nabla I_{p}^{\perp}$ is the isophote vector (where $\perp$ denotes the orthogonal operator). $D_{(p)}$ represents the strength of isophotes hitting the front $\delta \Omega$ at each iteration. Wherever, $\alpha$ is the normalization factor. Data term guarantees that the picture is inpainted following by the isophotes, which will make sure the linear structure is first filling. Fig. 2 show the details of $D_{(p)}$.

\section{B. Select the best matching patch}

This step mainly does searching work whose aim is to find the best matching patch $\varphi_{\hat{q}}$ in the source region for the target patch $\varphi_{p}$ whose priority is the highest. Equation (6) is the mearsurement of the similarity between each patch $\varphi_{q}$ in the source region and the target patch $\varphi_{p}$ :

$$
\begin{gathered}
\varphi_{\hat{q}}=\arg \min _{\varphi_{q} \cap \Phi} \mathrm{d}\left(\varphi_{\hat{p}}, \varphi_{q}\right) \\
d_{S S D}\left(\varphi_{p}, \varphi_{q}\right)=\sum\left[\begin{array}{c}
\left(R_{\varphi_{p}}-R_{\varphi_{q}}\right)^{2}+ \\
\left(G_{\varphi_{p}}-G_{\varphi_{q}}\right)^{2}+\left(B_{\varphi_{p}}-B_{\varphi_{q}}\right)^{2}
\end{array}\right]
\end{gathered}
$$

The distance $\mathrm{d}\left(\varphi_{\hat{p}}, \varphi_{q}\right)$ is defined as the sum of squared differences (SSD) of the known pixels in the two patches. The details is shown in equation (7) where $R, G$ and $B$ represents the value of intensity of each color channel.

\section{Inpaint the unknown area and update the confidence term}

In this step, the algorithm fills the unknown area by putting the best matching patch $\varphi_{\hat{q}}$ to the target patch $\varphi_{p}$ in the corresponding region. After that, we need to renew the boundary of the target region $\delta \Omega$ and update the confidence term using the following equation:

$$
C(q)=C(p), \forall q \in \varphi_{p} \cap \Omega
$$

Then, just continue to run the above steps until the unknown region is fully inpainted.

\section{PROPOSED METHOD}

In this section, the new update function for confident term and a new searching method are proposed.

\section{A. Confidence term updating}

Updating confidence term is an important step in Criminisi algorithm. Once the patch $\varphi_{p}$ is filled, the unknown pixel becomes a known point which will result the confidence value changing. Criminisi algorithm simply uses the best matching patch to inpaingting $\varphi_{p}$, and replace confidence term of the center pixel $p$ with the corresponding pixel's confidence. It is equivalent to using the function $f(x)=x, x \in[0,1]$ to update the confidence value. Along with the iterative process progresses, the confidence value will rapidly decrease and tend to zero, which may result in an incorrect inpainting direction.

In order to suppress the decay of confidence term in traditional methods, this paper proposes a new confidence update function. We suppose the function $h(x)$ as the following:

$$
h(x)=\log _{2}(x+1)
$$

Using the improved confidence function, the update equation used in the confidence update phase will be the following:

Where $\forall \mathrm{q} \in \varphi_{p} \cap \Omega$.

$$
C(q)=h(C(p))=\log _{2}(C(p)+1)
$$




\section{B. The new matching method}

The Criminisi algorithm uses SSD criteria in the matching processing to determine the similarity between sample blocks. Just calculate the sum of the squares difference of all known pixels in the block, and then select a target with the smallest error from the current repaired sample block as the best match in the source region. The matching formula is

$$
\varphi_{\hat{q}}=\arg \min _{\varphi_{q} \cap \Phi} \mathrm{d}\left(\varphi_{\hat{p}}, \varphi_{q}\right)
$$

Since the matching principle is too singular, and only the color feature is considered. When adopt the global search method, multiple optimal sample blocks may appear at a distance from the area to be repaired. Then the algorithm randomly selects one as the matching patch. Because the algorithm is a serial repair, the error copy of a patch will affect the subsequent match, which is easy to cause error accumulation and the quality of repair is degraded.

In an image, it can be divided into several regions according to different structural features. For a general candidate to be matched, the matching block should appear in the same or similar region as its own structure. And the probability that the matching block appears in other regions is small. Sometimes even if such a situation occurs, it may be a mismatch. This is because the information of the adjacent space is highly correlated, and the pixels that are far apart are less correlated, so the probability that the matching patch appears in the neighborhood is the largest. In order to minimize the probability of mismatching, we proposes an improved patch matching principle that incorporates the distance between the candidate patch center point and the target patch center point into the original similarity measure function.

When searching for matching patchs, we still choose global search. Because global search can overcome the shortcomings of local search's greedy character. So it can select the better matching patch to the greatest extent, and the target block inpaint can achieve global optimization. In the global search, we combine the SSD and physical distance for the calculation. The new matching formula is as follows:

$$
d_{\text {new }}\left(\varphi_{p}, \varphi_{q}\right)=d_{S S D}\left(\varphi_{p}, \varphi_{q}\right) * e^{\operatorname{dis}(p, q)}
$$

In our new calculation method, we combine SSD and physical distance to find the optimal matching block. In order to avoid that the physical distance is zero, we use the exponential form of the physical distance to calculate. The experimental results show that the new matching criterion significantly reduces the false matching rate, and the repair results are more in line with the visual connectivity of the human eye.

\section{EXPERIMENT AND ANALYSIS}

In the section, we test the proposed method on a few images with various background. Compared with the classical and stateof-art methods mentioned above, there are some improvements that can be seen from our results. Furth ermore, we set the same size for the square patch $\varphi_{p}$ in the experiments for Criminisi's method, Deng's method and the proposed method for the fairness.

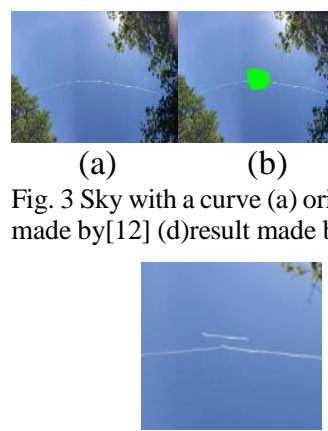

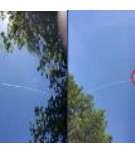

b)

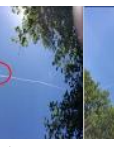

(c)

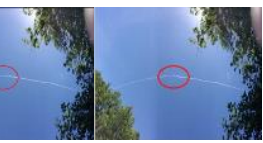

(d) (e)
Fig. 3 Sky with a curve (a) original image (b) region need to inpainting (c)result (a)

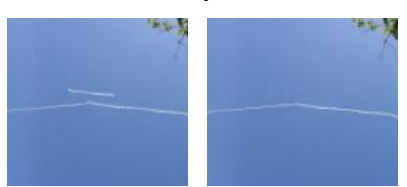

(b)

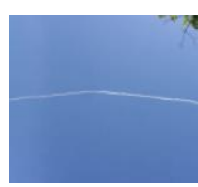

(c)
Fig. 4 Inpainting details of Fig. 3 (a)result made by[12] (b)result made by [23] (c)result made by proposed method

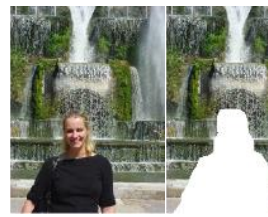

(a) (b)

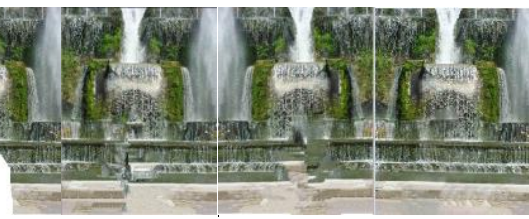

(c) (e) (d)
Fig. 5 Remove the girl(a) original image (b) region need to inpainting (c)result made by[12] (d)result made by [23] (e) result made by proposed

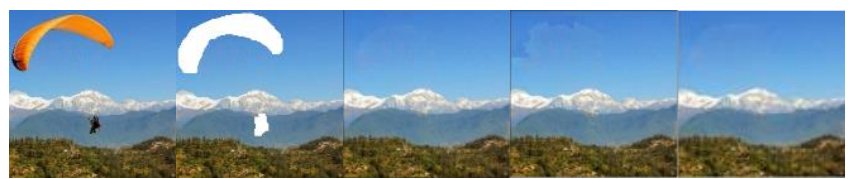

(c) (d) (e)
Fig. 6 Remove the Paragliding (a) original image (b) region need to inpainting (c)result made by[12] (d)result made by [23] (e) result made by proposed method

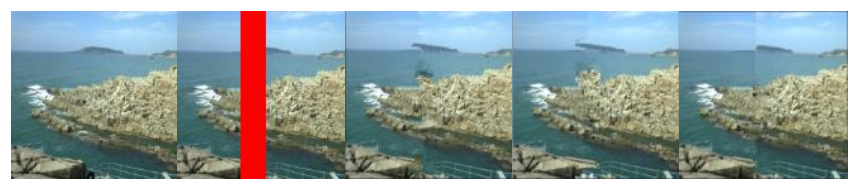

(c)

(d)

(e)

Fig. 7 Inpaint the red area in the sea(a) original image (b) region need to inpainting (c)result made by[12] (d)result made by [23] (e) result made by proposed method

Fig. 3 is the repair of a sky image. Obviously, a short line is emerged on the upper side of the curve in the result of Criminisi's method. Both Deng's algorithm and our algorithm avoid such mis-inpainting, and the results are more in line with real-life performance and human vision. The details of the inpainting results are shown in Fig. 4, which is more vivid to observation. In detail of the results, our method is much smoother than Deng's in terms of visual effects.

Fig. 5 is the image used in the Criminisi's paper. As can be seen from the results, the algorithm proposed by us has been greatly improved. Both competitive in terms of structure and texture. At the same time, we can see the effectiveness of the new algorithm in the subsequent of PSNR value. Fig. 6 is a target object removal for an image. The results are not clearly distinguishable. For this, we can use PSNR to judge. In the PSNR comparison in Table 1, we can observe that their values have not much difference, but there are still some. It can be seen that our algorithm is relatively better. 
Fig. 7 is a representation of a seaside picture of a selected area. As can be seen from the results, the method we proposed on the repair of the coast is the best, and the results of the other two methods have spit out. Our results look smoother and more in line with real-life coastal conditions. Our method did not show particularly good results in the inpaint of the reef in the middle of the sea. In addition, in the three inpaint results, we are very clear to see the occurrence of fault phenomenon. This shows that we still have a lot of research space to solve the problem.

PSNR (peak signal-to-noise ratio) is an objective standard widely used for evaluating the quality of image processing. In order to evaluate the effect of the inpainted image, the PSNR value is usually used to measure the inpainting performance of the algorithm. The larger the PSNR value is, the smaller the degree of image distortion. Thus, the better the algorithm inpainting performance. PSNR is calculated as:

$$
\mathrm{PSNR}=10 \log \left\{\frac{255^{2}}{\frac{1}{M \times N} \sum_{i=1}^{M} \sum_{j=1}^{N}[f(i, j)-\hat{f}(i . j)]^{2}}\right\}
$$

This paper also uses the PSNR to evaluate the effect of inpainting performance. Some PSNR values are shown in Table 1. It can be seen from Table 1 that the image inpainting methods proposed in this paper can obtain better results for images of different demaged regions. Since the structure and texture of the area to be repaired in Fig. 7 are relatively simple, the PSNR values of their results are similar regardless of the method. For images with more complex textures, as shown in Fig 6 and 8, the difference in PSNR values will be larger. Criminisi algorithm has difficulty in determining the confidence in the priority calculation and it is difficult to search for the best matching block, so that the PSNR value of the algorithm result is the lowest. Although Deng's algorithm improves the priority calculation, there is still problems in selection of the best matching block, which makes the PSNR value of the algorithm's result middle. Our method not only improves the confidence update, the selection of the best matching patch is strengthened. So, the PSNR value of our method gets the highest.

\begin{tabular}{cccc}
\hline TABLE I. & \multicolumn{3}{c}{ Comparison of PSNR Value for different methods } \\
\hline & Criminisi & Deng & ours \\
\hline Sky & 44.6362 & 46.9447 & 47.6480 \\
Girl & 13.8362 & 13.9845 & 14.0606 \\
Paragliding & 17.1514 & 17.1829 & 17.1924 \\
Sea & 25.7422 & 24.2435 & 26.9701 \\
\hline
\end{tabular}

\section{CONCLUSION}

This paper proposes a new confidence update function and the new matching method. The new confidence update function can effectively reduce the phenomenon that the confidence attenuation is too fast, and improve the accuracy of the image guidance repair direction. At the same time, combining the physical distance between blocks and traditional SSD matching criterion as the new matching method effectively improves the matching accuracy. The proposed method obtains competitive results when handle pictures with strong structure or texture compared with other state-of-the-art inpainting methods. Nevertheless, our method performed not so well when the picture including both strong structure and texture.

\section{ACKNOWLEDGMENT}

This research is partially supported by National Natural Science Foundation of China (No.61876018).

\section{REFERENCES}

[1] P. Buyssens, M. Daisy, D. Tschumperle, and O. Lezoray. Exemplar-based inpainting: Technical review and new heuristics for better geometric reconstructions. IEEE Trans. Image Processing (TIP), 24(6), 2015

[2] C. Guillemot and O. Le Meur, "Image inpainting: Overview and recent advances," IEEE Signal Process. Mag., pp. 127-144, Jan. 2014

[3] Chan T.F, Shen J. Mathematical models for local nontexture inpainting. SIAM Journal of Applied Mathematics. 2002; 62:1019-1043.

[4] Freeman W.T, Jones T.R, Pasztor E.C. Exemplar-based super-resolution. IEEE Computer Graphics and Applications. 2002; 22:56-65.

[5] Liang L, Liu C, Xu Y, Guo B, Shum H.Y. Real-time texture synthesis using patch-based sampling. ACM Trans on Graphics. 2001; 20:127-150.

[6] A. Efros and T. Leung, "Texture synthesis by non-parametric sampling," in Proc. Int. Conf. Computer Vision (ICCV), Sept. 1999, pp. 1033-1038.

[7] Bertalmio M, Sapiro G, Caselles V, Ballester C. Image inpainting. Proc ACM SIGGRAPH Computer Graphics (SIGGRAPH). 2000; p. 417-424.

[8] Chan T, Shen J (2001) Local inpainting models and tv inpainting. SIAM J Appl Math 62(3):1019-1043.

[9] Chan T, Shen J (2001) Non-texture inpainting by curvature-driven diffusions. J Vis Commun Image Represent 4(12):436-449.

[10] Telea A (2004) An image in-painting technique based on the fast marching method. J Graph Tools 9(1):23-34

[11] Criminisi A, Perez P, Toyama K. Object removal by exemplar-based image inpainting. Proc IEEE Conference on Computer Vision and Pattern Recognition (CVPR). 2003; p. 721-728.

[12] Criminisi A, Perez P, Toyama K. Region filling and object removal by exemplar-based image inpainting. IEEE Trans Image Processing. 2004; 13:1200-1212

[13] Qian Fan, Lifeng Zhang. A novel patch matching algorithm for exemplarbased image inpainting. Multimed Tools Appl(2017)

[14] Xu Z, Sun J. Image inpainting by patch propagation using patch sparsity. IEEE Trans Image Processing. 2010; 19:1153-1165

[15] Liu and V. Caselles, "Exemplar-based image inpainting using multiscale graph cuts," IEEE Trans. Image Process., vol. 22, no. 5,pp. 1699-1711.

[16] N. Komodakis and G. Tziritas, "Image completion using efficient belief propagation via priority scheduling and dynamic pruning," IEEE Trans. Image Process., vol. 16, no. 11, pp. 2649-2661, Nov. 2007.

[17] J. Mukherjee, and S. K. D. Mandal, "Image inpainting through metric labeling via guided patch mixing,” IEEE Trans. Image Process., vol. 25, no. 11, pp. 5212-5226, Nov. 2016.

[18] Barnes C, Shechtman E, Finkelstein A, Goldman D.B. PatchMatch: a randomized correspondence algorithm for structural image editing. ACM Transactions on Graphics (TOG). 2009; 28

[19] T. Ruzic and A. Pizurica, "Context-aware patch-based image inpainting using Markov random field modeling," IEEE Trans. Image Process., vol. 24, no. 1, pp. 444-456, Jan. 2015.

[20] W.H. Cheng, et al., Robust algorithm for exemplar-based image inpainting, The International Conference on Computer Graphics, Imaging and Vision(CGIV 2005), 2005, pp. , 64-69

[21] Q Fan, H Liu, Z Fu, X Li, Exemplar-Based Image Inpainting Based on Pixel Inhomogeneity Factor; Proceedings of APSIPA Annual Summit and Conference 2017, 12 - 15 December 2017, Malaysia

[22] J. Huang, S. Kang, N. Ahuja, J. Kopf, Image completion using planar structure guidance, ACM Trans. Graphics 33 (4) (2014) 129. github.com/jbhuang0604/StructCompletion

[23] Deng L-J, Huang T-Z, Zhao X-L (2015), "Exemplar-Based Image Inpainting Using a Modified Priority Definition." PLoS ONE 10(10): e014119 